\title{
A matrix isolation study of the fluorescence of anthracene and anthracene-ammonia adducts in solid argon
}

\author{
R. Fraenkel, U. Samuni, Y. Haas \\ Department of Physical Chemistry and the Farkas Center for Light Induced Processes, \\ The Hebrew University of Jerusalem, Jerusalem, Israel
}

and

B. Dick

Max Planck Institute of Biophysical Chemistry, W-3400 Göttingen, Germany

Received 19 November 1992

\begin{abstract}
The UV absorption and emission spectra of anthracene in an argon matrix were measured. Three major spectroscopic systems were observed, assignable to anthracene molecules occupying distinct sites. The origins of these systems were red-shifted with respect to the isolated molecule by 535,691 and $722 \mathrm{~cm}^{-1}$. At $17 \mathrm{~K}$ all lines were well resolved, with about $8 \mathrm{~cm}^{-1}$ fwhm. Addition of a small amount of ammonia leads to the observation of three new band systems, red-shifted with respect to the previously mentioned ones. These observations are discussed in relation to recent studies of anthracene-argon and anthracene-ammonia clusters in supersonic jets.
\end{abstract}

\section{Introduction}

The UV spectroscopy of anthracene has been extensively studied. The first observable strong transition has its origin at $27695 \mathrm{~cm}^{-1}$ in the gas phase $[1,2]$, and is assigned to the ${ }^{1} \mathrm{~L}_{\mathbf{a}} \leftarrow{ }^{1} \mathrm{~A}$ transition (oscillator strength $\approx 0.1$ ). In the $D_{2 h}$ point symmetry group the transition is labeled as ${ }^{1} \mathrm{~B}_{2 \mathrm{u}}^{+} \leftarrow{ }^{1} \mathrm{~A}_{\mathrm{lg}}$. In the usual convention [3], $y$ is the long in-plane axis and $x$ the out-of-plane axis and all vibrational bands of the transition belong either to the $a_{g}$ or to the $b_{3 g}$ symmetries. High-resolution studies were also performed in Shpolskii matrices [4,5] and in frozen solutions [6]. Argon matrices were studied in relation to analytical applications - it was found that narrow lines could be obtained, and that different polycyclic aromatic hydrocarbon molecules could be simultaneously determined [7]. In these studies, no details were given as to the spectroscopic properties of the matrix-isolated molecules, such as the number of distinct sites, the exact shifts with respect to the gas phase, linewidths, etc.
Recently, several studies on the solvation of anthracene (and other aromatic molecules) by rare-gas atoms in supersonic jets were reported. It was found that the solvent shift usually increased with the cluster size, but also appeared to depend on cluster geometry [8,9]. Hayes et al. [10] reported that in a large excess of argon, a broad $\left(\approx 200 \mathrm{~cm}^{-1}\right)$ band was observed at about $26930 \mathrm{~cm}^{-1}$, namely a shift of $\approx 760 \mathrm{~cm}^{-1}$ to the red with respect to the bare molecule. This band was assigned tentatively to large clusters, possibly mimicking bulk liquid or solid. Amirav et al. [11] found, in a somewhat different experimental setup, a shift of $-610 \mathrm{~cm}^{-1}$ and a width of $97 \mathrm{~cm}^{-1}$. In several cases, the solvent shift was indeed found to extrapolate to the bulk value [11]. However, it was recently pointed out [12] that this is not always the case, although it is not yet possible to choose among different possible causes for this observation. A semi-empirical theoretical calculation of the shift [13] was found to reproduce reasonably well the experimental results of many systems, with the notable exception of anthracene. It is 
thus of interest to explore the details of the solvation behaviour of anthracene in a rare-gas matrix, by establishing the relevant spectroscopic parameters. This might help to account for discrepancies such as found between previous reports (e.g. refs. $[10,11]$ ) and to establish a better connection between matrix and cluster work.

Some time ago, we studied the anthracene-ammonia system in a supersonic jet [14]. It was found that two distinct fluorescence systems were present: one consisted of a series of narrow bands, similar in nature to the anthracene-rare gas adducts. The fluorescence decay time of these bands was about $10^{-8}$ s. A second system, characterized by much broader excitation and emission bands, and by a decay time of a few hundred nanoseconds, was also observed. It was assigned to a charge-transfer exciplex system. Similar bands were observed in the past for anthracene, or other aromatic hydrocarbon molecules, with aromatic amines, such as dimethylaniline [15]. Another goal of this work was to obtain further information on the anthracene-ammonia adducts by conducting the experiments in a cold matrix.

\section{Experimental}

Matrices were prepared by depositing a rare gas, saturated by anthracene vapour by passing it over crystals held at room temperature (estimated vapour pressure $10^{-5}$ Torr [16]), onto a $\mathrm{BaF}_{2}$ window. The deposition rate was $12 \mathrm{mmol} / \mathrm{h}$ and the argon/anthracene ratio was estimated to be $1: 10^{-5}$. The window was cooled to the desired temperature by an Air Products cryostat (model CS202K). When samples containing ammonia were prepared, the ammonia-rare gas mixture was premixed in the desired ratio prior to passing it over the crystals. $\mathrm{Ab}$ sorption spectra were taken with a mercury-xenon arc as the light source, combined with a $3 / 4 \mathrm{~m}$ monochromator (Spex 1701). Fluorescence excitation was by a tunable dye laser (Quanta Ray model PDL-1), pumped by an Nd:YAG laser. In Jerusalem, emission was dispersed by the same monochromator as used in the light absorption experiments and detected by a photomultiplier tube (Hamamatsu R329) whose output was fed into a digital oscilloscope. The signal was averaged and processed by a personal computer. In Göttingen, the experimental setup consisted of a Leybold cryostat (model ROK 300/10), a 0.85 m monochromator (Spex 1402). Excitation was by a Lambda Physik (FL3002) dye laser, pumped by an excimer laser (Lambda Physik EMG101) and detection was done by a CCD camera, allowing simultaneous recording of the fluorescence and emission spectra.

Analytical grade anthracene was used throughout the experiments. In a few cases, zone refined samples were used as a control, with no distinguishable difference. Ammonia (Matheson 99.99\%) was used as received, as was high-purity argon (Matheson $99.9995 \%)$.

\section{Results}

A small portion of the absorption spectrum of anthracene in an argon matrix at $17 \mathrm{~K}$ is shown in fig. 1. It is seen that three distinct bands are observed. Tuning the laser to any one of them, led to strong emission which, upon dispersion, was found to consist of three different, distinguishable fluorescence band systems. A more careful survey showed that

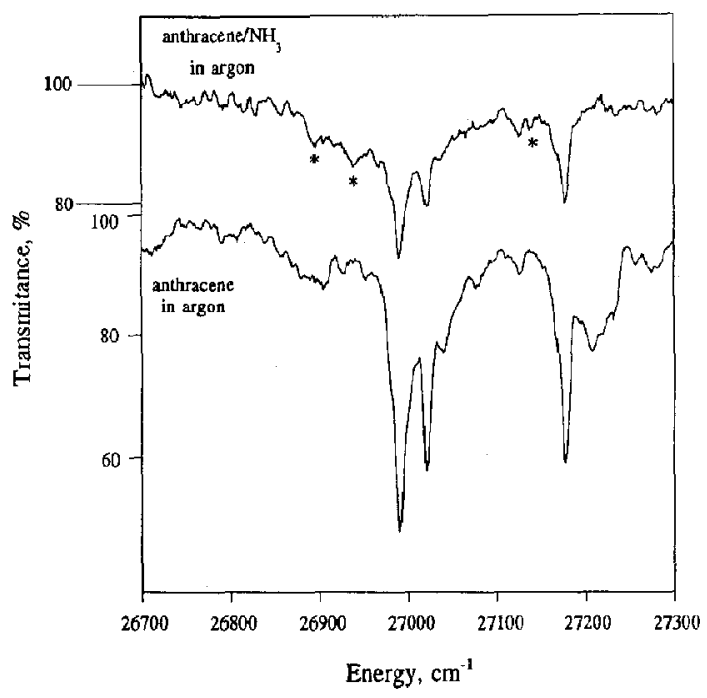

Fig. 1. The absorption spectrum of anthracene in argon at about $370 \mathrm{~nm}$ (lower trace) and in the presence of ammonia (upper trace). The three main absorption lines are due to the origins of the three main sites. The bands due to anthracene-ammonia adducts are marked by asterisks. 
some further band systems were present, whose intensity was much smaller than the main three and are not discussed further in this Letter. The two-dimensional plot shown in fig. 2 demonstrates clearly how the different sites are manifested in the spectrum (see also fig. 3). The origins of the three main systems were at 27159,27004 and $26973 \mathrm{~cm}^{-1}$. In the following, the respective sites will be referred to as 1,2 and 3. A listing of the positions of the main fluorescence bands, along with their assignment is presented in table 1 . The assignments are based on previous fluorescence [1,4-6,18], Raman [19] and theoretical [17] work, and arc discussed further in section 4 . The widths of the bands, in both emission and excitation, was found to be $8 \pm 1 \mathrm{~cm}^{-1}$. The instrumental resolution was $0.2 \mathrm{~cm}^{-1}$ in excitation, and $3 \mathrm{~cm}^{-1}$ in emission; thus the width is due to an intrinsic property such as inhomogeneities in the sites, with a possible contribution to broadening due to anthracene-argon vibrations. The emission decay time was not measured, since it was found to be of the same order as the laser pulse duration $(20 \mathrm{nsec})$ or shorter. In some cases, a phonon band due to one site overlapped a zero phonon band of another site, causing the appearance of extra lines in the emission spectrum. These lines are not listed in table 1.

Adding a small amount of ammonia to the argon gas before passing it oyer the anthracene crystals, resulted in the appearance of new bands in the spectrum. Again, three distinct major band systems could be clearly discerned; their origins were found to be at 27111,26919 and $26973 \mathrm{~cm}^{-1}$ and are depicted in fig. 1. These systems are assigned to new sites, which will be referred to as $1 \mathrm{~A}, 2 \mathrm{~A}$ and $3 \mathrm{~A}$, respectively. Due to the small concentration of the anthracene-ammonia adducts, the exact location of the origin bands, and the other transitions were more accurately determined by fluorescence spectroscopy.

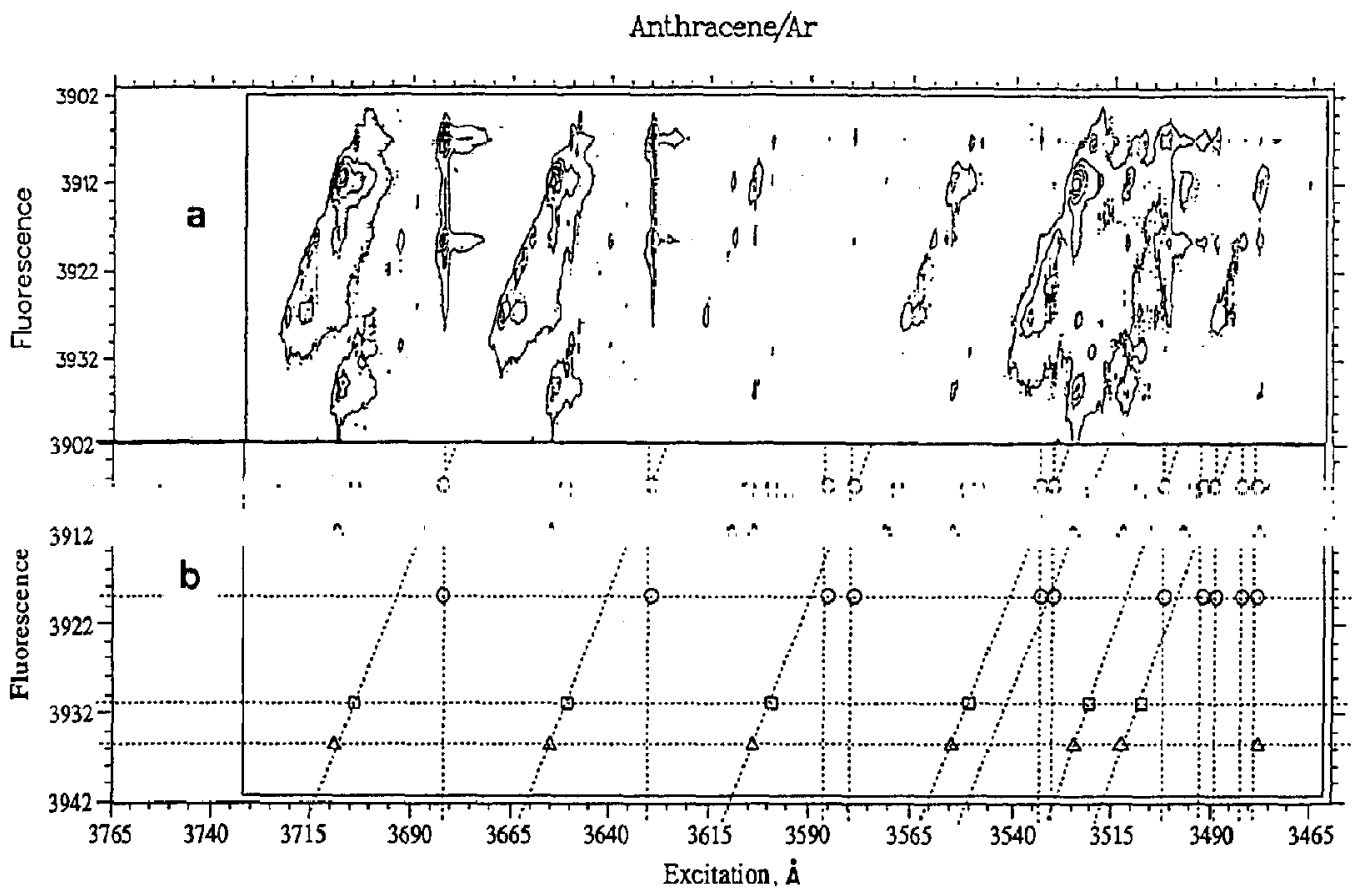

Fig. 2. (a) A two-dimensional plot of the fluorescence intensity of anthracene in an argon matrix as a function of both excitation and emission wavelengths. One can clearly distinguish between different sites by observing the regularity along diagonals. Vertical cuts yield fluorescence spectra at a given excitation frequency, and horizontal ones yield excitation spectra at a given emission frequency. Examples of the latter are shown in fig. 3. (b) Analyzed contour plot. Only zero phonon lines of the bands' vibronic transitions are shown. They are marked by a different graphical shape. Bands of the same transition, from all sites, are along a diagonal line. Fluorescence emission bands of each site are located along a vertical line. (Marked here only for one site.) 
Anthracene/Ar

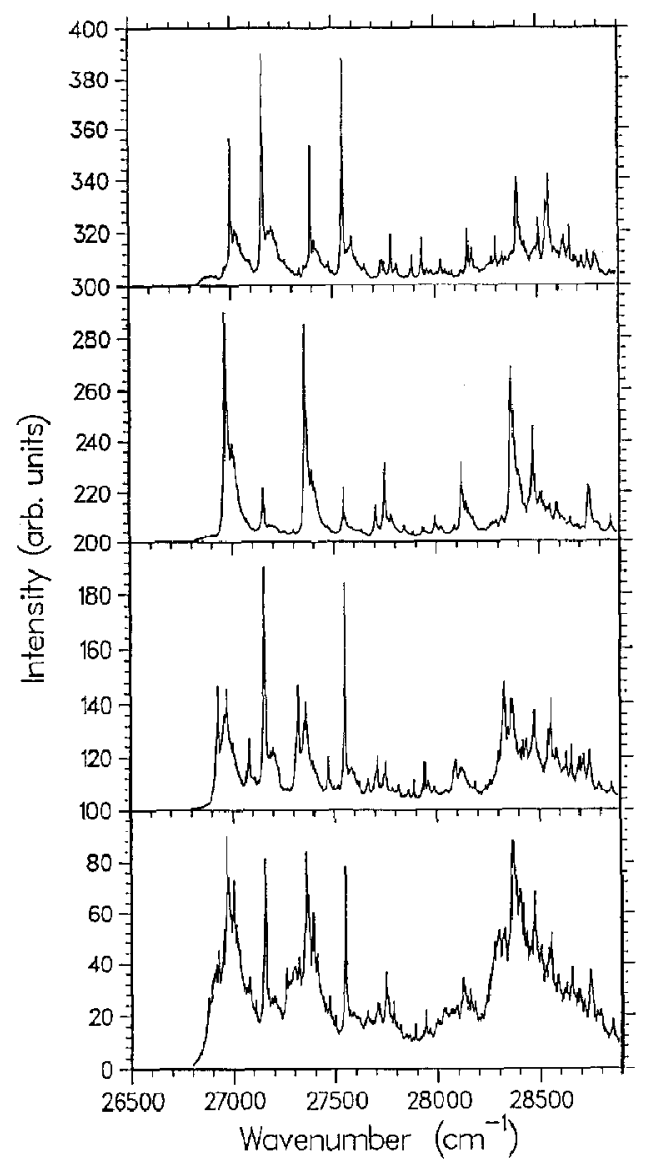

Fig. 3. Cuts across the spectrum shown in fig. 2a, showing separately the excitation spectra observed upon monitoring the emission at $3907 \AA$ (top trace), $3911.5 \AA$ (second from top), 3918.5 $\AA$ (third trace), and the broad band excitation spectrum obtained upon combining the three (bottom trace). The latter corresponds approximately to the absorption spectrum.

Fig. 4 shows emission spectra of anthracene in an argon matrix containing ammonia, showing three spectroscopic series. Two belong to anthracene sites, and one to an anthracene-ammonia site. The frequency intervals observed, as seen from the figure and from table 1, are within experimental error, those characteristic of anthracene.

In the presence of ammonia, another emission band was also observed; its amplitude was much smaller than that of the three main bands and its width much larger. The emission spectrum was cen- tered around $580 \mathrm{~nm}$, namely shifted considerably to the red from the emission discussed above, whose major bands are between 380 and $420 \mathrm{~nm}$. The excitation band extended from $370 \mathrm{~nm}$, where it overlapped with the narrow lines, to at least $1000 \mathrm{~cm}^{-1}$ to the red of the 0,0 band. It could not be accurately determined, since the monochromator slits had to be opened to $1 \mathrm{~mm}$ ( $11 \AA$ resolution) to obtain the data. The decay time of the broad emission band was 1.56 $\mu \mathrm{sec}$ - much longer than that of the narrow bands. It could not be observed in the absence of either anthracene or ammonia. This system is assigned to a charge transfer transition and is further discussed in a separate communication [20].

\section{Data analysis}

The appearance of several band systems in the spectrum of a molecule embedded in a low-temperature matrix can arise from the co-existence of several sites, or of different conformers. The exact correspondence between the line spacings obtained by us in all band systems and the line spacings observed in other low-temperature environments [4-6,18,21], in the gas phase $[1,2]$ and for the ground state by Raman spectroscopy [19] is a clear indication that these systems are all due to anthracene molecules in somewhat modified environments. In the matrix, this corresponds to different sites.

The conditions used in this work - low temperatures and a high spectroscopic resolution - are required in order to distinguish between different sites. Even so, the exact location of a given band involves a tedious iterative procedure, when the excitation and emission spectra are separately scanned. The use of two-dimensional plotting of the fluorescence intensity as a function of both excitation and emission frequencies makes the location of sites and the discrimination between zero phonon lines and phonon wings a straightforward matter. The method, also termed total luminescence spectroscopy, has been used extensively in the past (see, for instance, ref. [22]). In our case, appropriate cuts across the two-dimen. sional surface allowed the immediate determination of a band system belonging to a given site. 
Table 1

Ground state vibrational frequencies from the observed transitions in the fluorescence spectrum of anthracene in an argon matrix, and in an argon matrix with ammonia added. Only main sites are shown. Line origins and intervals are given in $\mathrm{cm}^{-1 \text { a) }}$

\begin{tabular}{|c|c|c|c|c|c|c|c|c|}
\hline \multirow[t]{3}{*}{ Assignment ${ }^{\text {d }}$} & \multirow[t]{3}{*}{ Gas $^{b)}$} & \multicolumn{6}{|c|}{ Matrix sites $^{e)}$} & \multirow[t]{3}{*}{ Intensity } \\
\hline & & \multicolumn{3}{|c|}{ anthracene } & \multicolumn{3}{|c|}{ anthracene $/ \mathrm{NH}_{3}$} & \\
\hline & & 1 & 2 & 3 & $1 \mathrm{~A}$ & $2 \mathrm{~A}$ & $3 \mathrm{~A}$ & \\
\hline \multirow[t]{2}{*}{ Sl origin } & 27691 & 27159 & 27004 & 26973 & 27111 & 26919 & 26890 & \\
\hline & 237 & - & 235 & 236 & 240 & 226 & 239 & $\mathrm{vw}$ \\
\hline 12 & 390 & 388 & 390 & 387 & 390 & 389 & 395 & $\mathrm{~s}$ \\
\hline 11 & 624 & 623 & 628 & 625 & 623 & 626 & - & $\mathbf{w}$ \\
\hline 10 & 753 & 756 & 753 & 757 & 756 & 757 & 756 & $\mathbf{w}$ \\
\hline $2 * 12$ & 778 & 791 & 787 & 784 & 791 & 784 & 797 & $\mathrm{~m}$ \\
\hline 21 & 912 & 915 & 911 & 915 & 914 & 912 & 912 & vw \\
\hline 9 & 1012 & 1011 & 1005 & 1008 & 1010 & 1005 & 1013 & vw \\
\hline 8 & 1165 & 1161 & 1167 & 1163 & 1159 & 1168 & 1172 & $\mathrm{~m}$ \\
\hline \multirow[t]{2}{*}{19} & 1183 & 1180 & 1180 & - & 1180 & 1190 & 1192 & vw \\
\hline & 1226 & 1235 & 1227 & 1236 & 1233 & - & - & $\mathrm{vw}$ \\
\hline 18 & 1250 & 1248 & 1253 & 1256 & 1254 & - & - & vw \\
\hline 7 & 1263 & 1262 & 1266 & 1269 & 1267 & 1260 & 1271 & $\mathbf{m}$ \\
\hline \multirow[t]{2}{*}{$12+21$} & 1304 & - & 1299 & (?) 1315 & 1307 & (?) 1300 & - & vw \\
\hline & 1382 & (?) 1336 & 1339 & 1341 & - & $(?) 1359$ & - & $w$ \\
\hline \multirow[t]{2}{*}{6} & 1408 & 1415 & 1411 & 1407 & 1406 & 1411 & 1421 & $\mathbf{s}$ \\
\hline & 1519 & 1508 & - & 1511 & - & 1507 & - & $v w$ \\
\hline 4 & 1566 & 1567 & 1561 & 1563 & 1564 & 1560 & 1576 & $\mathbf{m}$ \\
\hline \multirow[t]{2}{*}{15} & 1643 & 1639 & 1638 & 1634 & 1642 & 1637 & 1646 & $\mathrm{~m}$ \\
\hline & 1654 & - & - & - & - & 1656 & - & vw \\
\hline $6+12$ & 1797 & 1800 & 1798 & 1799 & 1803 & 1802 & 1811 & $\mathrm{~m}$ \\
\hline $4+12$ & & 1954 & 1962 & 1957 & 1956 & 1959 & 1974 & $w$ \\
\hline $12+15$ & & 2030 & 2037 & 2032 & 2032 & 2033 & 2035 & $\mathbf{w}$ \\
\hline $6+10$ & & 2168 & 2162 & 2161 & 2163 & 2169 & - & vw \\
\hline $6+2 * 12$ & & 2193 & 2186 & 2186 & 2194 & 2187 & 2183 & $\mathbf{w}$ \\
\hline 278 & & 2348 & 2333 & 2327 & 2342 & 2351 & 2341 & vw \\
\hline $6+9$ & & 2428 & 2430 & 2418 & 2422 & 2429 & 2430 & $w$ \\
\hline $6+8$ & & 2574 & 2574 & 2573 & 2574 & 2572 & 2579 & $\mathbf{w}$ \\
\hline $6+7$ & & 2670 & 2675 & 2674 & 2669 & 2673 & 2672 & $\mathrm{w}$ \\
\hline $4+8$ & & 2730 & 2728 & 2733 & 2729 & 2726 & 2725 & vw \\
\hline \multirow[t]{2}{*}{$2^{*} 6$} & & 2819 & 2817 & 2815 & 2818 & 2813 & 2825 & $\mathrm{~m}$ \\
\hline & & - & - & 2891 & - & 2894 & 2894 & vw \\
\hline $6+8+12$ & & 2966 & 2968 & 2966 & 2964 & 2969 & 2980 & $\mathrm{~m}$ \\
\hline 3 & & 3042 & 3043 & 3041 & 3040 & 3049 & 3048 & w \\
\hline $2 * 4$ & & 3123 & 3128 & 3126 & 3121 & 3123 & - & $\mathbf{v w}$ \\
\hline \multirow{2}{*}{$\left.\begin{array}{l}2^{*} 6+12 \\
4+15\end{array}\right\}$} & & 3210 & 3213 & 3206 & 3207 & 3207 & 3218 & $\mathbf{w}$ \\
\hline & & - & - & 3346 & - & 3346 & - & vw \\
\hline $4+6+12$ & & 3363 & 3360 & 3357 & 3360 & 3358 & 3358 & vw \\
\hline $6+12+15$ & & 3437 & 3432 & 3429 & 3433 & 3435 & 3433 & vw \\
\hline
\end{tabular}

a) Relative frequencies accurate to within $\pm 6 \mathrm{~cm}^{-1}$.

b) Ref. [1].

c) Line positions less certain due to unfavourable $\mathrm{S} / \mathrm{N}$ ratio are marked with (?).

d) Ref. [17]. The numbering of the vibrational levels follows Krainov's work. Levels 1-12 belong to $A_{1 \mathfrak{q}}$ symmetry, and levels 13-22 belong to $B_{18}$ symmetry. 


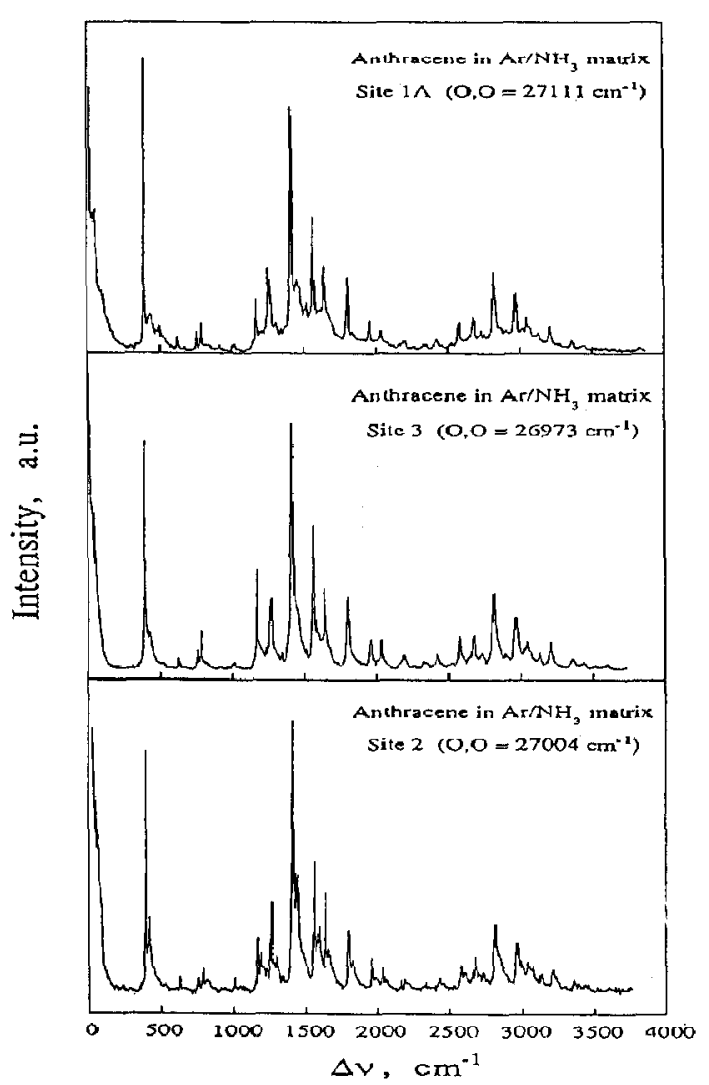

Fig. 4. A plot of the fluorescence spectra of anthracene in an argon $/ \mathrm{NH}_{3}$ matrix upon excitation of the 0,0 transition. The two lower traces show the spectra obtained upon excitation of isolated anthracene in sites 2 and 3 , at $370.31 \mathrm{~nm}$ (bottom) and $370.74 \mathrm{~nm}$ (middle). The top trace shows the spectrum of an anthracene-ammonia site, $1 \mathrm{~A}$, excited at $368.85 \mathrm{~nm} . \Delta \nu$ is the frequency above the zero-point energy.

\section{Discussion}

\subsection{Interaction between anthracene and ammonia}

Since we find that addition of ammonia also leads to the appearance of only three major sites, it is reasonable to assume that they correspond to the original three sites found in an argon matrix, by replacing an argon atom (or several) with an ammonia molecule. In the jet, the $1: 1$ adducts exhibit a shift of 41 [8] or 48 [9] $\mathrm{cm}^{-1}$ for one argon atom, and $95 \mathrm{~cm}^{-1}$ for one ammonia molecule [15]. The sites appearing upon adding ammonia are seen to be shifted as compared to the pure anthracene sites by $46 \mathrm{~cm}^{-1}$ for site 1 , and by $86 \mathrm{~cm}^{-1}$ for sites 2 and
3 (table 1). Invoking the principle of additive shifts, a possible interpretation is that in site 1 one ammonia molecule replaces an argon atom in the first solvation layer of anthracene, and in sites 2 and 3, two ammonia molecules are replacing argon atoms in that layer. Another possibility is that one ammonia molecule replaces one argon atom, at different locations next to the anthracene molecule, causing different shifts. This model is not supported by available jet cluster data.

In these types of sites, the interaction between the components is weak compared to intramolecular forces, and therefore the spectrum of the $\pi-\pi^{*}$ transition maintains its vibrational structure, and is only somewhat red-shifted. In contrast, the low-amplitude broad band that correlates well with a corresponding weak and broad transition observed in the supersonic jet [14], is assigned to a charge transfer transition $[14,15]$, representing a much stronger interaction between anthracene and ammonia.

\subsection{Relation to cluster work}

Stepwise solvation by argon and other rare-gas atoms in a supersonic jet was reported by several groups. In small clusters, the solvent shift is always to the red, and roughly linear with the number of raregas atoms attached to anthracene $[1,2,23]$. The linewidth of each transition can be made as narrow as that of the isolated molecule $\left(1-2 \mathrm{~cm}^{-1}\right)$ by using appropriate stagnation conditions, and is determined by the rotational envelope and the laser linewidth.

In order to observe large clusters, a high pressure of the rare gas is required (up to several atmospheres [10]) and the appearance of the spectrum changes: rather than a series of narrow lines, the spectrum consists now of rather broad - up to several hundred $\mathrm{cm}^{-1}$ - features. The appearance of such broad bands has been assigned $[10,24]$ to several reasons: different shifts of structural isomers or of different sized clusters and insufficient cooling due to the heat released upon the condensation process during the formation of large clusters. Under these conditions transitions originating from vibrationally and rotationally excited clusters contribute to the observed spectrum, thus leading to the appearance of broad features. 
In the matrix a similar situation corresponds to the high-temperature case, in which phonon bands dominate the spectrum. Our experiments show that, if the system is properly cooled, narrow lines can be made to dominate the spectrum. However, since we find three major sites and several minor ones, one should also consider that at the limit of infinite cluster size $[11,24]$, several conformations should be considered. We note that the red-shifts found in this work - 532, 687 and $718 \mathrm{~cm}^{-1}$ - cover the range of shifts found for large anthracene clusters in jets $[10,11]$. By finding the suitable cooling conditions in the jet, one may observe a convergence of the series to one of three matrix sites or, perhaps, to all of them.

\subsection{Conclusion and comparison with other environments}

Using narrow-band laser excitation and low temperatures has allowed the observation of highly resolved, zero phonon transitions of large aromatic molecules. The results of some such measurements for the 0,0 transitions are summarized in table 2 . The range of shifts is rather small - it is only about twice as big for typical organic solvents as for raregas atoms. In several cases more than one 0,0 band is found, indicating the co-existence of several sites of similar stabilization energies. The high-temperature spectra are dominated by phonon bands, leading to broad features, in which only the most intense vibronic transitions can still be discerned. The solvent shift remains essentially the same as in the low temperatures.

The results presented in this work support the notion that if the interaction between solvent molecules and the solute is weak, clusters with a relatively small number of solvent molecules can indeed serve as a bridge between the isolated molecule and the solvated one [26]. The co-existence of several distinct sites of similar stability must be considered when extrapolating cluster properties to infinitely large systems.

\section{Acknowledgement}

The Farkas Center for Light Induced Processes is
Table 2

The 0,0 transitions of the first $\pi-\pi^{*}$ transition in anthracene

\begin{tabular}{|c|c|c|c|}
\hline Medium & $\begin{array}{l}\text { Transition } \\
\text { frequency } \\
\left(\mathrm{cm}^{-1}\right)\end{array}$ & Shift & Ref. \\
\hline gas & 27691 & 0 & {$[1]$} \\
\hline Ar matrix & $\begin{array}{l}27159 \\
27004 \\
26973\end{array}$ & $\begin{array}{l}532 \\
687 \\
718\end{array}$ & $\begin{array}{l}\text { this work } \\
\text { this work } \\
\text { this work }\end{array}$ \\
\hline $\begin{array}{l}\text { Ar/ammonia } \\
\text { matrix }\end{array}$ & $\begin{array}{l}27111 \\
26919 \\
26890\end{array}$ & $\begin{array}{l}580 \\
772 \\
801\end{array}$ & $\begin{array}{l}\text { this work } \\
\text { this work } \\
\text { this work }\end{array}$ \\
\hline hexane/cyclohexane & 26525 & 1166 & [4] \\
\hline hexane & $\begin{array}{l}26487 \\
26447 \\
26323\end{array}$ & $\begin{array}{l}1204 \\
1244 \\
1368\end{array}$ & $\begin{array}{l}{[21]} \\
{[21]} \\
{[21]}\end{array}$ \\
\hline$n$-octane/cyclohexane & 26455 & 1235 & [4] \\
\hline \multirow[t]{4}{*}{ heptane } & $\begin{array}{l}26402 \\
26264 \\
26218\end{array}$ & $\begin{array}{l}1289 \\
1427 \\
1473\end{array}$ & $\begin{array}{l}{[21]} \\
{[21]} \\
{[21]}\end{array}$ \\
\hline & $\begin{array}{l}26283 \\
26265 \\
26232\end{array}$ & $\begin{array}{l}1408 \\
1426 \\
1459\end{array}$ & $\begin{array}{l}{[18]} \\
{[18]} \\
{[18]}\end{array}$ \\
\hline & 26227 & 1464 & [6] \\
\hline & $\begin{array}{l}26221 \\
26246\end{array}$ & $\begin{array}{l}1470 \\
1445\end{array}$ & $\begin{array}{l}{[25]} \\
{[25]}\end{array}$ \\
\hline octane & $\begin{array}{l}26630 \\
26600 \\
26161\end{array}$ & $\begin{array}{l}1061 \\
1091 \\
1530\end{array}$ & $\begin{array}{l}{[21]} \\
{[21]} \\
{[21]}\end{array}$ \\
\hline biphenyl & 26056 & 1635 & {$[25]$} \\
\hline \multirow[t]{2}{*}{ fluorene } & 25988 & 1703 & {$[5]$} \\
\hline & 25975 & 1716 & [25] \\
\hline
\end{tabular}

supported by the Minerva Society for Research, mbH, Munich, Germany.

\section{References}

[1] W.R. Lambert, P.M. Felker, J.A. Syage and A.H. Zewail, J. Chem. Phys. 81 (1984) 2195; W.R, Lambert, P.M. Felker and A.H. Zewail, J. Chem. Phys. 81 (1984) 2209.

[2] A. Amirav, C. Horwitz and J. Jortner, J. Chem. Phys. 88 (1988) 3092.

[3] R.S. Mulliken, J. Chem. Phys. 23 (1955) 1997.

[4] G.F. Kirkbright and C.G. de Lima, Analyst 99 (1974) 333. 
[5] T. Tam and P. Saari, Chem. Phys. 40 (1979) 311.

[6] T.P. Carter and G.D. Gillespie, J. Phys. Chem. 86 (1982) 2691.

[ 7] G. Mamantov, E.L. Wehry, R.R. Kremer and E.R. Hinton, Anal. Chem. 49 (1977) 86.

[8] A. Amirav, U. Even and J. Jortner, Chem. Phys. Letters 72 (1980) 16.

[9] W.E. Henke, W. Yu, H.L. Selzle, E.W. Schlag, D. Wutz and S.H. Lin, Chem. Phys. 92 (1985) 187.

[10] T.R. Hayes, W.E. Henke, H.L. Selzle and E.W. Schlag, Chem. Phys. Letters 77 (1981) 19; Z. Naturforsch. (1980) 1429.

[11] A. Amirav, U. Even and J. Jortner, J. Phys. Chem. 86 (1982) 3345.

[12] X. Li, M.Y. Hahn, M.L. El-Shall and R.L. Whetten, J. Phys. Chem. 95 (1991) 8524.

[13] E. Shalev, N. Ben-Horin, U. Even and J. Jortner, J. Chem. Phys. 95 ( 1991 ) 3147.

[14] O. Anner and Y. Haas, J. Phys. Chem. 90 (1986) 4298.

[15] Y. Haas and $O$. Anner, in: Photoinduced electron transfer, eds. M.A. Fox and M. Chanon (Elsevier, Amsterdam, 1988) p. 305.
[16] A.B. Macnick and J.M. Prausnitz, J. Chem. Eng. Data 24 (1979) 175.

[17] E.P. Krainov, Opt. Spectry. 16 (1964) 532;

D.J. Evans and D.B. Scully, Spectrochim. Acta 20 (1964) 891;

K. Ohno, J. Mol. Spectry. 77 (1979) 329.

[18 ] J.L. Richards and S.A. Rice, J. Chem. Phys. 54 (1971) 2014

[19] J. Rasanen, F. Stenman and E. Penttinen, Spectrochim. Acta 29A (1973) 395.

[20] R. Fraenkel and Y. Haas, J. Phys. Chem., in press.

[21] R.M. Macnab and K. Sauer, J. Chem. Phys. 53 (1970) 2805.

[22] K. Palewska, E.C. Meister and U.P. Wild, J. Luminescence 50 (1991) 47.

[23] A. Heikal, L. Banares, D.H. Semmes and A.H. Zewail, Chem. Phys. 157 (1991) 231.

[24] J. Jortner, Ber. Bunsenges. Phys. Chem. 88 (1988) 188; S. Leutweiler and J. Jortner, Faraday Discussions Chem. Soc. 86 (1988) paper 19 ;

J. Bösiger, R. Bombach and S. Leutweiler, J. Chem. Phys. 94 (1991) 5098.

[25] A.V. Bree and S. Katagiri, J. Mol. Spectry. 17 (1965) 24.

[26] C. Crepin and A. Tramer, Chem. Phys. Letters 170 (1990) 446. 